Received 00th January 20xx,

Accepted 00th January 20xx DOI: $10.1039 / x 0 \times x 00000 x$

\section{Nanoengineering multifunctional hybrid interfaces using adhesive}

\section{glycogen nanoparticles}

\author{
Pietro Pacchin Tomanin ${ }^{a}$, Jiajing Zhou ${ }^{a}$, Alessia Amodio ${ }^{a}$, Rita Cimino ${ }^{a}$, Agata Glaba , Francesca \\ Cavalieri $^{\mathrm{b}, \mathrm{c}, *}$, Frank Caruso ${ }^{\mathrm{a}, *}$
}

\begin{abstract}
Multifunctional and biodegradable nanostructured hybrid interfaces based on biopolymers are potentially useful in many applications in catalysis, bioanalytical sensing and nanomedicine. Herein, we report the engineering of multifunctional hybrid films by assembling adhesive biological nanoparticles i.e. lipoate-conjugated phytoglycogen (L-PG). These nano building blocks possess adhesive properties, arising from their amphiphilic nature, and reactive functional disulfide groups. The assembly of L-PG on surfaces enabled the rapid and conformal deposition of a thin film on substrates of varying chemical composition and wettability. The L-PG films showed negligible cytotoxicity and moderate stability under different harsh conditions but displayed enzyme-mediated degradability. In addition, metal nanoparticles were embedded into the L-PG layers to build up multilayered hybrid films. Specifically, Au and Ag nanoparticle-loaded L-PG multilayered films with catalytic and surface-enhanced Raman scattering properties were prepared. Finally, we highlight the versatility of the present approach to engineer multifaceted interfaces for catalysis and sensing applications.
\end{abstract}

\section{Introduction}

The engineering of multifunctional nanostructured interfaces has attracted great interest in many interdisciplinary fields including nanomedicine, ${ }^{1}$ biosensing, ${ }^{2}$ tissue engineering, ${ }^{3}$ and catalysis. ${ }^{4}$ The desired multifunctional interface can be achieved by controlling the chemical functionality and structural arrangement of the building blocks on a nanometer scale. For integration of functions, a polymeric material with the desired functionality and morphology can be combined with inorganic or metal nanoparticles to engineer a complex hybrid interface with structural and compositional inhomogeneity..$^{5-7}$ The precise manipulation of the physical and chemical interactions between these hybrid building blocks allows control of the interface nanomorphology and functionality of the macroscopic materials. The use of polysaccharides, such as chitosan, $^{8.9}$ amylose, $^{10}$ cellulose, ${ }^{11}$ hyaluronic acid, ${ }^{12,13}$ as biodegradable polymeric components for engineering multifunctional surfaces and films, is attractive because

\footnotetext{
a. ARC Centre of Excellence in Convergent Bio-Nano Science and Technology, and the Department of Chemical Engineering, The University of Melbourne, Parkville Victoria 3010, Australia. E-mail: fcaruso@unimelb.edu.au

${ }^{b}$ School of Science, RMIT University, Melbourne, Victoria 3000, Australia. E-mail: francesca.cavalieri@rmit.edu.au

'Dipartimento di Scienze e Tecnologie Chimiche, Università di Roma "Tor Vergata', via della ricerca scientifica 1, 00133, Rome, Italy. E-mail: francesca.cavalieri@rmit.edu.au

Electronic Supplementary Information (ESI) available: Synthesis of L-PG, DD-PG and AuNP, details on experimental procedures and materials characterization. See DOI: $10.1039 / x 0 x x 00000 x$
}

polysaccharides are natural biomacromolecules that can be chemically or biochemically modified to impart various functional properties. In particular, glycogen is a biological polysaccharidic nanoparticle found ubiquitously across the animal and plant kingdoms, serving as a vital component of the cellular energy machinery. ${ }^{14,15}$ Glycogen nanoparticles have a dendrimer-like structure composed of repeating units of glucose connected by linear $\alpha-(1,4)$ glycosidic linkages with $\alpha-(1,6)$ branching. They have different structural properties (e.g., nanoparticle size, molecular weight, and degree of branching) depending on the source. ${ }^{16}$ For example, phytoglycogen (PG) is a naturally abundant form of glycogen derived from sweet corn, possessing a diameter of $80 \mathrm{~nm}$ and a high molecular weight of $20 \mathrm{MDa} .{ }^{16} \mathrm{PG}$ nanoparticles have a highly branched, hydrated structure ${ }^{17,18}$ and a high colloidal stability, and chemical functionalization of the polymeric chains can be performed under mild conditions using nontoxic reagents. Importantly, the reactive hydroxyl groups on glycogen can be modified to produce functional nanoparticles that remain partially biodegradable, ${ }^{19}$ making glycogen attractive as a building block for engineering biofunctional materials and interfaces. Recently, glycogen nanoparticles with different chemical modifications and properties have been successfully exploited for drug delivery, ${ }^{20-23}$ targeting, ${ }^{24}$ cellular imaging, ${ }^{19,25}$ and smart materials. ${ }^{26-29}$

Amine functionalities are typically introduced on glycogen to form complexes with negatively charged nucleic acids. ${ }^{19-20}$ Octenylsuccinate hydroxypropyl PG nanoparticles were developed to improve the solubility of hydrophobic dugs. ${ }^{29}$ The modification of glycogen with hydrophobic allyl and propargyl moieties was 
exploited to obtain crosslinked water-insoluble films with a fibrous or sponge-like structure. The films were used as a scaffold for growing osteoblast-like cells. ${ }^{30} \mathrm{~A}$ stimuli-responsive hydrogel for colon-targeted drug delivery was obtained via free-radical polymerization of glycogen, modified by $\mathrm{N}$-isopropylacrylamide moieties, with ethylene glycol dimethacrylate as a crosslinker. ${ }^{31}$ Despite the various glycogen-based materials developed to date, the use of the adhesive properties of glycogen nanoparticles for the preparation of functional interfaces remains to be examined and exploited. The development of adhesive glycogen nanoparticles and self-assembly strategies is expected to advance this biological nanoparticle toward its use as a versatile surface coating material. We propose that the modification of PG with hydrophobic and functional moieties would endow the functionalized amphiphilic polysaccharide with unexplored adhesive properties to prepare nontoxic, biodegradable films.

In the present study, we report a rapid and robust method to form nanostructured hybrid films using lipoate-conjugated phytoglycogen (L-PG). L-PG exhibits remarkable adhesiveness to coat various substrates including organic, inorganic, and even lowsurface-energy materials (e.g., polytetrafluoroethylene (PTFE)). A thin and homogenous film is rapidly formed by immersion of the substrate into an L-PG water solution. The resulting L-PG film shows negligible cytotoxicity and significant degradability, yet displays stability in various harsh environments such as strong acid. We also demonstrate that these films can be functionalized with inorganic nanoparticles (e.g., Au, Ag, and Pt nanoparticles) owing to the high affinity of disulfide bridges in the tagged lipoate to the noble metal nanoparticles. The supported $\mathrm{Au}$ nanoparticles (AuNPs) show catalytic activity and high stability owing to the high permeability and protection of the L-PG multilayered film. Moreover, a surfaceenhanced Raman scattering (SERS) active substrate is developed by integrating $\mathrm{Ag}$ nanoparticles (AgNPs) into the coating, therefore producing sensing interfaces that can monitor chemical changes in the surface proximity. Overall, this study describes a rapid and facile strategy for preparing multifunctional and biodegradable interfaces for different applications including in the environmental and medical fields.

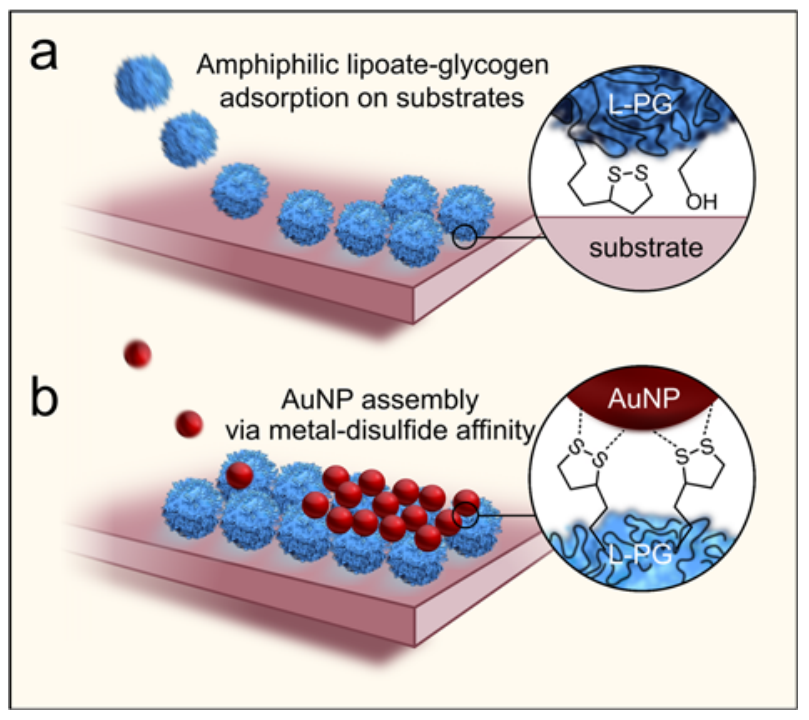

Fig. 1. (a) Schematic of the L-PG assembly on a substrate and (b) coassembly of metal nanoparticles on the L-PG layer to obtain hybrid surfaces via LbL assembly suitable for catalysis and sensing applications.

\section{Experimental}

\section{Materials}

Phytoglycogen derived from sweet corn was purchased from Glysantis. Acetic acid glacial, anhydrous dimethyl sulfoxide (DMSO) ( $\geq 99.9 \%$ ), ammonium molybdate, $\beta$-amylase from barley, copper(II) sulfate pentahydrate, crystal violet (CV), deuterium oxide, dialysis tubing cellulose membrane (14 kDa cutoff), fetal bovine serum, glutathione (GSH), gold(III) chloride hydrate, hydrochloric acid, lipoic acid (LA), methylene blue, nitric acid, Parafilm $M$, phosphate buffered saline (PBS) tablets, platinum nanopowder $(<50 \mathrm{~nm})$, polyethylene terephthalate (PET) film, polymethylmethacrylate (PMMA) film, polypropylene (PP) film, polystyrene (PS) film, PTFE film, AgNPs $(20 \mathrm{~nm})$, sodium acetate, sodium arsenate dibasic pentahydrate, sodium bicarbonate, sodium borohydride, sodium carbonate, sodium citrate, sodium dodecyl sulfate (SDS), sodium potassium tartrate tetrahydrate, sodium sulfate, sulfuric acid, thiazolyl blue tetrazolium bromide (MTT), and $\mathrm{N}$-(3dimethylaminopropyl)- $N^{\prime}$-ethylcarbodiimide hydrochloride (EDC.HCl) were purchased from Sigma-Aldrich. Glass substrates were purchased from Knittel Glass. PMMA substrates were purchased from Caplugs Evergreen. 4-(Dimethylamino)pyridine (DMAP) was purchased from Fluka. Alexa Fluor 488 NHS Ester (AF488) and Dulbecco's modified Eagle medium were purchased from Thermo Fisher Scientific. Dodecanoic acid was purchased from Alfa Aesar. Hydrogen peroxide, ethanol (EtOH), and $n$-hexane were purchased from Chem-Supply. A PS microparticle dispersion $(3.2 \mu \mathrm{m})$ was purchased from MicroParticles. PP tubes $(15 \mathrm{~mL})$ were purchased from Corning. Nickel foam was purchased from American Elements. PS cuvettes were purchased from Greiner Bio-One. PTFE and polyurethane tubes were purchased from ATA Scientific. All the chemicals were used as received. High-purity (Milli-Q) water with a resistivity of $18.2 \mathrm{M} \Omega \mathrm{cm}$ was obtained from an inline Millipore RiOs/origin water purification system.

\section{Synthesis of L-PG}

To modify PG with a degree of functionalization of $2 \%$ (LA/glucose $\mathrm{mol} / \mathrm{mol}$ ), PG (200 mg, $1.23 \mathrm{mmol}$ glucose unit) was dissolved in $8 \mathrm{~mL}$ anhydrous DMSO under an inert atmosphere of nitrogen. LA (30.6 $\mathrm{mg}, 0.12$ eq.), DMAP ( $45.2 \mathrm{mg}, 0.3$ eq.), and EDC.HCl (28.4 mg, 0.12 eq.), each dissolved in $4 \mathrm{~mL}$ anhydrous DMSO, were sequentially added dropwise to the above solution under stirring. The system was allowed to react for $72 \mathrm{~h}$ under nitrogen gas flux. Then, $\mathrm{HCl}$ (1.2 M, $0.31 \mathrm{~mL}, 0.3$ eq.) was slowly added to neutralize DMAP. The product was purified by dialysis ( $14 \mathrm{kDa}$ cutoff) against acidic Milli-Q water ( 2 $\mathrm{L}, \mathrm{pH} 4$ using $\mathrm{HCl}$ ) for 2 days and neutral Milli-Q water for one day, and freeze-dried to finally obtain L-PG (yield $180 \mathrm{mg}, 90 \%$ ). The degree of functionalization of glycogen was determined by NMR spectroscopy. ${ }^{1} \mathrm{H}-\mathrm{NMR}\left(400 \mathrm{MHz}, \mathrm{D}_{2} \mathrm{O}, 50^{\circ} \mathrm{C}\right) \delta(\mathrm{ppm}): 5.36(0.98, \mathrm{H}-$ $\mathrm{a \alpha}), 4.97(0.02, \mathrm{H}-\mathrm{a} \beta), 4.30-3.32(6.53, \mathrm{H}-\mathrm{b}, \mathrm{c}, \mathrm{d}, \mathrm{e}, \mathrm{f}), 3.31-3.20$ (0.02, H-k, m), 2.62-2.4 (0.07, H-g), 2.08-1.94 (0.03, H-I), 1.87-1.19 
(0.13, $\mathrm{H}-\mathrm{h}, \mathrm{i}, \mathrm{j})$. The degree of functionalization was calculated by dividing the peaks integral relative to the lipoate peaks $(\delta(\mathrm{ppm})$ : 3.31-1.19) by the peaks integral of $\mathrm{H}-\mathrm{a} \alpha$ and $\mathrm{H}-\mathrm{a} \beta$ ( $\delta(\mathrm{ppm})$ : 5.60 $4.80)$ to 100.

\section{Preparation of L-PG coatings}

To prepare L-PG coatings, substrates were incubated in a $0.1 \mathrm{mg} \mathrm{mL}^{-1}$ L-PG water solution for $30 \mathrm{~min}$ at $21^{\circ} \mathrm{C}$ and then rinsed with water to remove unbound polymer. The film was dried by nitrogen flow. Freshly prepared samples were used for subsequent characterizations.

\section{Preparation of L-PG metal nanoparticle hybrid coatings on glass}

Circular glass substrates (diameter of $10 \mathrm{~mm}$ ) were functionalized with L-PG and Au, Ag and Pt metal nanoparticles. First, the substrates were cleaned with $\mathrm{EtOH}$ and water and placed on a planar surface. To prepare the first layer, an L-PG dispersion $\left(0.4 \mathrm{~mL}, 0.1 \mathrm{mg} \mathrm{mL}^{-1}\right)$ was deposited on the glass. After incubation for $10 \mathrm{~min}$, the glass was rinsed with water. To prepare the second layer of metal nanoparticles, $0.4 \mathrm{~mL}$ of a $\mathrm{Au}\left(0.06 \mathrm{mg} \mathrm{mL}^{-1}\right), \mathrm{Ag}\left(0.02 \mathrm{mg} \mathrm{mL}^{-1}\right)$, or Pt $\left(0.1 \mathrm{mg} \mathrm{mL}^{-1}\right)$ colloidal dispersion was added and the system was incubated for another $10 \mathrm{~min}$. The glass was then rinsed with water to obtain one bilayer on one side of the substrate. To prepare multilayered or multi-elements systems, the process was repeated as many times as required with the appropriate metal nanoparticles. When the appropriate number of bilayers was obtained, the glass was dried with a $\mathrm{N}_{2}$ gun. For control experiments, unmodified PG was used with AuNPs following the abovementioned procedure; and the glass substrates were directly incubated with AuNPs in the absence of L-PG following the same procedure.

\section{Preparation of PS microparticles coated with L-PG and AuNPs}

A dispersion of $3.2 \mu \mathrm{m}$ PS microparticles $\left(0.3 \mathrm{~mL}, 1 \mathrm{mg} \mathrm{mL}^{-1}\right)$ was washed via centrifugation and redispersion with water three times (2000 rcf, $2 \mathrm{~min}$ ). After each centrifugation, sonication in an ultrasound bath was performed for $5 \mathrm{~s}$ to disperse the particles in solution. During the last wash, the dispersion was diluted with water to $0.3 \mathrm{~mL}$ and added via pipetting to an L-PG dispersion $(0.2 \mathrm{~mL}, 0.1$

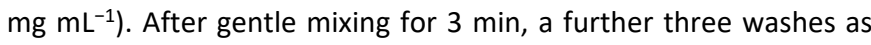
previously described were performed. In the last wash, the dispersion was diluted to $0.3 \mathrm{~mL}$ to obtain a $1 \mathrm{mg} \mathrm{mL}^{-1} \mathrm{~L}-\mathrm{PG}$-coated PS microparticle dispersion. To this dispersion, $0.5 \mathrm{~mL}$ of AuNP colloidal dispersion was added by pipetting and the solution was gently mixed for $3 \mathrm{~min}$. The colloidal dispersion was then washed three times as previously described and finally diluted to $0.3 \mathrm{~mL}$ to obtain a $1 \mathrm{mg} \mathrm{mL}^{-1}$ PS microparticles coated with one L-PG/AuNP bilayer. The assembly cycle was repeated as many times as required, with the last layer composed of AuNP after each cycle.

\section{Preparation of inner-coated PP tubes with L-PG and AuNPs}

To prepare an inner-coated $15 \mathrm{~mL}$ PP tube via layer-by-layer (LbL) assembly, an L-PG dispersion ( $2 \mathrm{~mL}, 0.1 \mathrm{mg} \mathrm{mL}^{-1}$ ) was introduced into the PP tube and vortexed for $3 \mathrm{~min}$, followed by washing with water. Then, a AuNP colloidal dispersion ( $2 \mathrm{~mL}, 0.06 \mathrm{mg} \mathrm{mL}^{-1}$ ) was introduced into the tube, vortexed for $3 \mathrm{~min}$, and washed with water, producing a 1-bilayer inner-coated PP tube. To obtain systems with more bilayers, the steps were repeated several times as required. The functionalized tubes were used fresh for the catalysis experiments.

\section{Characterization}

Attenuated total reflectance Fourier transform infrared (ATR-FTIR) spectra were obtained on a TENSOR II (Bruker) ATR-FTIR spectrometer. UV-vis absorbance spectra were recorded on a SPECORD 250 PLUS (Analytik Jena, Germany) spectrophotometer or an Infinite M200 microplate reader (Tecan, Switzerland). Dynamic light scattering (DLS) hydrodynamic diameter and $\zeta$-potential were measured using a Zetasizer Nano-ZS instrument (Malvern Instruments, Malvern, UK). Atomic force microscopy (AFM) experiments were performed on a JPK NanoWizard II BioAFM instrument. Contact angle experiments were performed on an optical contact angle tensiometer (DataPhysics) using the sessile drop method. Cell viability was evaluated via a standard MTT assay protocol using MDA-MB-231 cells. The rate of glycogen nanoparticle degradation by $\beta$-amylase was determined using the SomogyiNelson assay. The chemical stability of the L-PG coatings was studied via fluorescence analysis using a Fluorolog spectrofluorometer (Jobin Yvon Horiba). Quartz crystal microbalance with dissipation (QCM-D) experiments were performed using a QSense E4 (Biolin Scientific, Sweden) equipped with gold sensor chips (QSX 301 Gold, Biolin Scientific). Differential interference contrast (DIC) microscopy images were recorded using an inverted Olympus IX71 microscope. TEM images were acquired using a Tecnai Spirit (FEI, USA) instrument with an operation voltage of $120 \mathrm{kV}$. SEM images and EDX spectra and mapping were acquired with a FlexSEM 1000 (Hitachi, Japan) benchtop scanning electron microscope equipped with a Quantax 80 (Bruker, USA) EDX system. Helium ion microscopy images were recorded on an ORION NanoFab (Zeiss, Peabody, USA) using a $25 \mathrm{keV}$ $\mathrm{He}^{+}$probe with a current of $0.3 \mathrm{pA}$. SERS analysis was conducted on a RENISHAW Raman microscope equipped with a $\mathrm{He}-\mathrm{Ne}$ laser operating at $532 \mathrm{~nm}$.

\section{Results and Discussion}

\section{Synthesis and characterization of L-PG}

PG was first functionalized with a natural compound, LA, via an esterification reaction in DMSO to form L-PG (Figure S1). The resulting L-PG bears both hydrophilic and hydrophobic moieties (Figure 1a) that impart adhesion properties to the nanoparticles. In addition, the disulfide groups of lipoate are reactive toward metal nanoparticles (Figure 1b). NMR spectra confirmed the successful preparation of L-PG, which showed distinct signals that were ascribed to the glucose repeating unit and lipoate moieties (Figure S2). The lipoate groups are likely grafted to the outermost branches of PG. Previous work has shown that glycogen inner chains that are not swollen by organic solvent (e.g., DMF and DMSO) are less accessible and reactive, leading to surface-constrained functionalization. ${ }^{32}$ It is noteworthy that a trade-off between hydrophilicity and hydrophobicity is vital in the design of this building block. For example, samples with a degree of functionalization higher 


\section{ARTICLE}
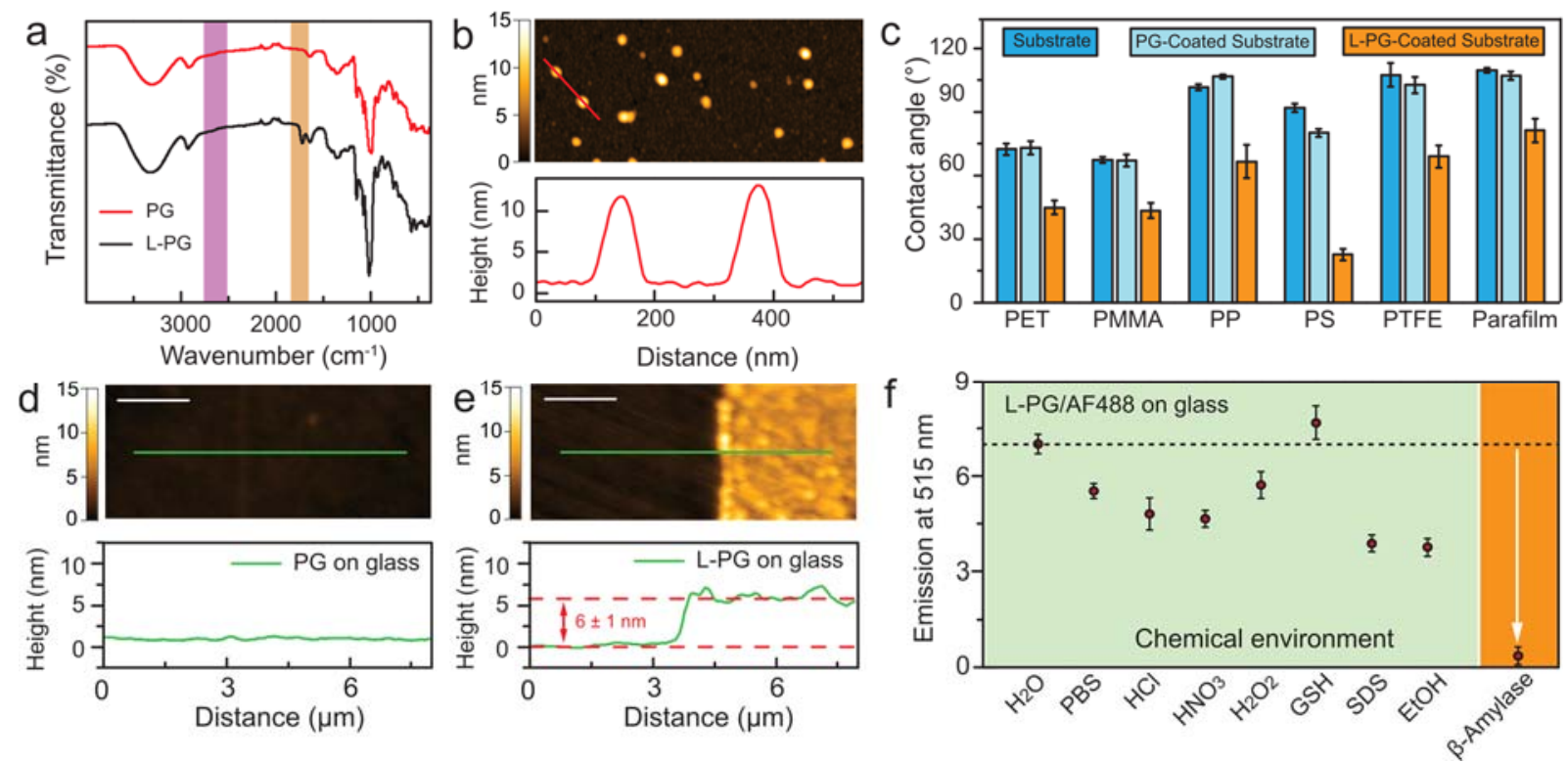

Fig. 2. Characterization and coating properties of L-PG. (a) ATR-FTIR spectra of PG and L-PG and (b) dry-state AFM image and corresponding height profile of L-PG. (c) Water contact angles of the bare, PG-, and L-PG-coated substrates. Dry-state AFM images and corresponding height profiles of (d) PG- and (e) L-PG-coated glass substrates (scale bar $2 \mu \mathrm{m}$ ). (f) Emission intensities of fluorescently labeled L-PG coatings on glass substrates after incubation in different chemical and biochemical environments. Error bars represent standard deviations.

than $2 \%$ showed poor solubility in water and DMSO owing to the high hydrophobicity (Table S1). Therefore, a degree of functionalization of $2 \%$ was considered optimal for maintaining L-PG colloidal stability in aqueous solution. The presence of lipoate groups on L-PG was further confirmed by FTIR spectroscopy. The newly formed ester bonds were observed at $1721 \mathrm{~cm}^{-1}$ (Figure 2a). Importantly, the 1,2dithiolane of lipoate remained in the oxidized state during the reaction, as signals corresponding to thiol stretching bands at 2560$2570 \mathrm{~cm}^{-1}$ were absent. ${ }^{33}$ The new absorbance peak observed at 290 $\mathrm{nm}$ in the UV-Vis spectrum of L-PG further confirmed the presence of the lipoate ring (Figure S3). ${ }^{34}$ The synthesized L-PG had a hydrodynamic diameter $(90 \pm 50 \mathrm{~nm})$, as determined by DLS, and a $\zeta-$ potential value $(-1 \pm 6 \mathrm{mV})$ similar to those of unmodified PG $(90 \pm$ $40 \mathrm{~nm} ;-1 \pm 6 \mathrm{mV}$ ) (Figure S4). These results confirm that the chemical modification of PG with up to $2 \%$ hydrophobic moieties does not induce any aggregation or substantial change in the solution properties of the resulting nanoparticles relative to those of the unmodified PG. Dry-state AFM further confirmed that the L-PG nanoparticles were spherical and well dispersed, with a round flattened shape, characteristic of dehydrated spherical soft materials (Figure 2b). As measured by AFM, the L-PG nanoparticles featured an average diameter of $90 \pm 20 \mathrm{~nm}$ and a thickness of $8 \pm 2 \mathrm{~nm}$.

\section{Preparation and characterization of L-PG coatings}

L-PG was subsequently examined for coating several planar polymeric substrates, including PET, PMMA, PP, PS, Parafilm, and PTFE. The successful coating was confirmed by contact angle measurements (Figure 2c). Specifically, the L-PG film formed on a PTFE surface featured a reduced water contact angle from $107 \pm 6^{\circ}$ (uncoated PTFE substrate) to $69 \pm 5^{\circ}$, thus shifting the wettability of the final film from hydrophobic to hydrophilic. A similar trend was observed on other substrates (Figures $2 \mathrm{c}$ and S5). After coating with L-PG, the substrates displayed a lower water contact angle compared to the bare substrates because the amphiphilic films exhibited a hydrophilic character. Conversely, when unmodified PG was used to coat these substrates, there was negligible difference in the contact angle before and after the deposition procedure (Figure $2 \mathrm{c}$ ). This observation indicates that the unmodified PG nanoparticles show poor adhesiveness. In addition, AFM analysis revealed that, unlike unmodified PG (Figure 2d), a continuous, dense L-PG film of $6 \pm 1 \mathrm{~nm}$ in thickness formed on glass after deposition (Figure 2e).

To examine the stability of the coatings, L-PG was labeled with a fluorophore tag (AF488) so that the disassembly of the coating was monitored after the treatment. The L-PG coating was first incubated in different solutions for $60 \mathrm{~min}$, followed by repeated washing with water. The fluorescence intensity of the substrates was then 
recorded to evaluate residual L-PG on the substrates. The results indicate that the L-PG coating shows moderate stability in diverse chemical environments such as in $\mathrm{H}_{2} \mathrm{O}, 10 \mathrm{mM}$ PBS ( $\mathrm{pH}$ 7.4), $1 \mathrm{M} \mathrm{HCl}$, $1 \mathrm{M} \mathrm{HNO}_{3}, 1 \mathrm{M} \mathrm{H}_{2} \mathrm{O}_{2}, 10 \mathrm{mM} \mathrm{GSH}, 17 \mathrm{mM} \mathrm{SDS}$, and EtOH (Figure 2f). In contrast, the L-PG film can be significantly degraded by $\beta$-amylase (Figure 2f), an exoenzyme that catalyzes the hydrolysis of glycosidic linkages from the non-reducing chain ends to release maltose. However, the L-PG nanoparticles required a longer incubation time than the unmodified PG nanoparticles to achieve the same degree of degradation by $\beta$-amylase (Figure S6). The L-PG film can act as depot for the sustained release of maltose in biological environments. Additionally, the released building blocks are not cytotoxic (Figure S7).

These results show that stable aqueous dispersions of amphiphilic L-PG nanoparticles can be used to build robust but biodegradable, noncytotoxic thin films owing to the adhesiveness of L-PG that promotes the interfacial assembly of nanoparticles on substrates with different wettability.

To understand the mechanism governing the adhesion properties of L-PG, the influence of the amphiphilic nature of PG without disulfide bonds was assessed. To this end, an analogous amphiphilic PG derivative, containing 1\% hydrophobic dodecane moiety (DD-PG) was synthesized (Figure S8). DD-PG displayed properties similar to those of L-PG such as hydrodynamic diameter $(71 \pm 40 \mathrm{~nm})$ and $\zeta$-potential $(-1 \pm 5 \mathrm{mV})$. The results showed that the water-soluble DD-PG also formed a film on glass similar to L-PG, with a similar thickness and surface morphology (Figure S9). These findings underline the importance of the amphiphilic characteristic of functionalized PG for the successful formation of coatings. Moreover, the disulfide moieties in L-PG offer an additional advantage for further functionalization of the L-PG films.

\section{Characterization of L-PG/metal nanoparticles hybrid films}

Herein, we demonstrate that glycogen-inorganic hybrid films with controlled thickness can be fabricated by LbL assembly of L-PG and metal nanoparticles, including AuNPs, AgNPs, and platinum nanoparticles (PtNPs) (Figure 3a). The assembly of the L-PG/AuNP film was monitored by measuring the (linear) increase in the intensity of the localized surface plasmon resonance (LSPR) band of the AuNPs at $545 \mathrm{~nm}$. (Figure S10). Notably, the narrow LSPR peak indicates the relatively large interparticle distance between neighboring AuNPs, suggesting that the AuNPs do not form clusters when embedded in the film and L-PG nanoparticles act as a spacer. Similar trends in relation to the increase in absorbance intensity were observed in the multilayered hybrid films containing AgNPs (LSPR at $415 \mathrm{~nm}$ ) or PtNPs (a broad absorbance profile spanning the UV-vis region without a characteristic peak) (Figure S11). A multicomponent hybrid film with three bilayers obtained by sequential deposition of L-PG/AuNP, L-PG/AgNP, and L-PG/PtNP is also demonstrated. The resulting UV-vis spectrum exhibited a combined absorption profile comprising the typical absorbance of each metal nanoparticle assembly (Figure 3a, red line). To demonstrate the importance of disulfide moieties in coordinating the metal NPs for the successful assembly of the hybrid films, control experiments were performed using DD-PG/AuNP, unmodified PG/AuNP, and AuNPs. The deposition of these control systems on glass resulted in very weak LSPR bands, indicating poor adsorption on the glass substrate (Figure $\mathrm{S} 12)$. These results highlight the importance of disulfide bonds on LPG for the successful loading of AuNPs.
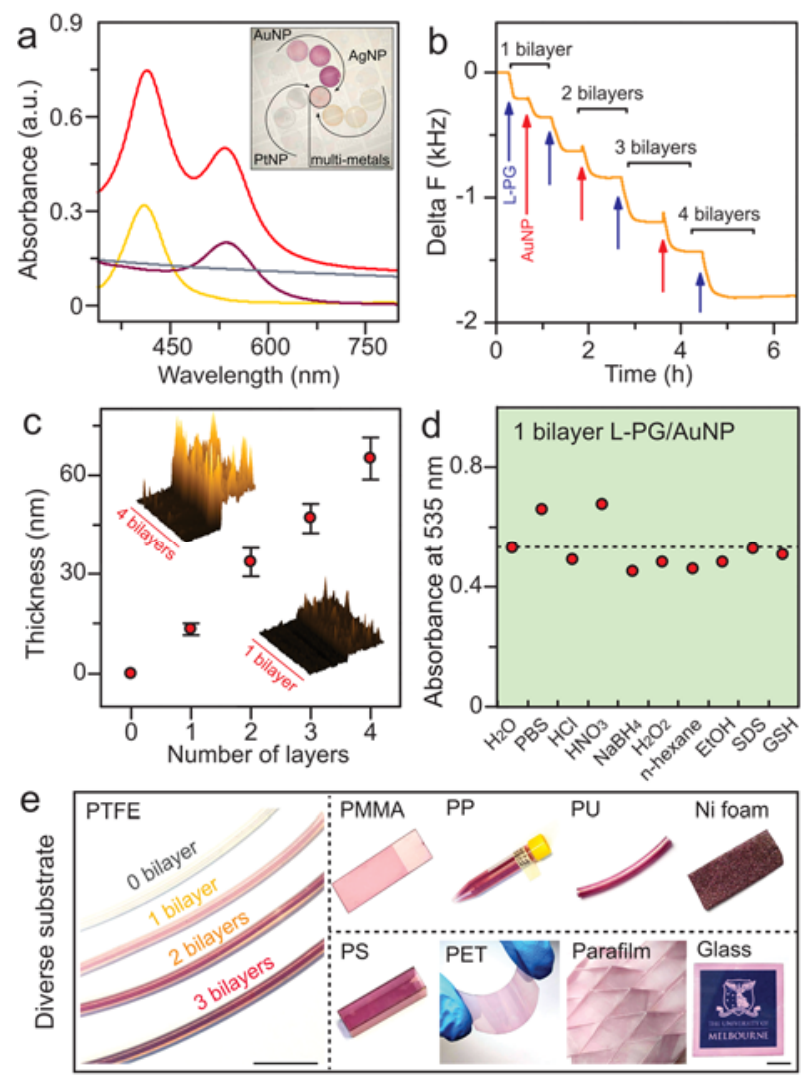

Fig. 3. Characterization of hybrid L-PG LbL assemblies with metal nanoparticles. (a) UV-Vis spectra of glass substrates coated with one bilayer of L-PG/AuNP, L-PG/AgNP and L-PG/PtNP (purple, yellow and grey lines, respectively) and three bilayers of sequentially assembled L-PG/AuNP, L-PG/AgNP, and L-PG/PtNP (red line) and corresponding photograph (inset) of glass substrates coated with various layers of L-PG and metal nanoparticles ( $\mathrm{Au}, \mathrm{Ag}, \mathrm{Pt}$, and $\mathrm{Au}-\mathrm{Ag}-\mathrm{Pt}$ ). (b) QCM-D frequency profile of an L-PG/AuNP LbL assembly on a gold chip obtained by sequential deposition of L-PG (blue arrow) and AuNPs (red arrow). (c) Dry-state AFM film thickness profile as a function of the L-PG/AuNP bilayer number and corresponding AFM images of the first and fourth bilayers (insets). (d) LSPR absorbance of glass substrates with 1 bilayer of L-PG/AuNPs after incubation in various chemical environments. (e) Photographs of L-PG/AuNP LbL assemblies on various substrates (scale bar $1 \mathrm{~cm}$ ). 


\section{ARTICLE}
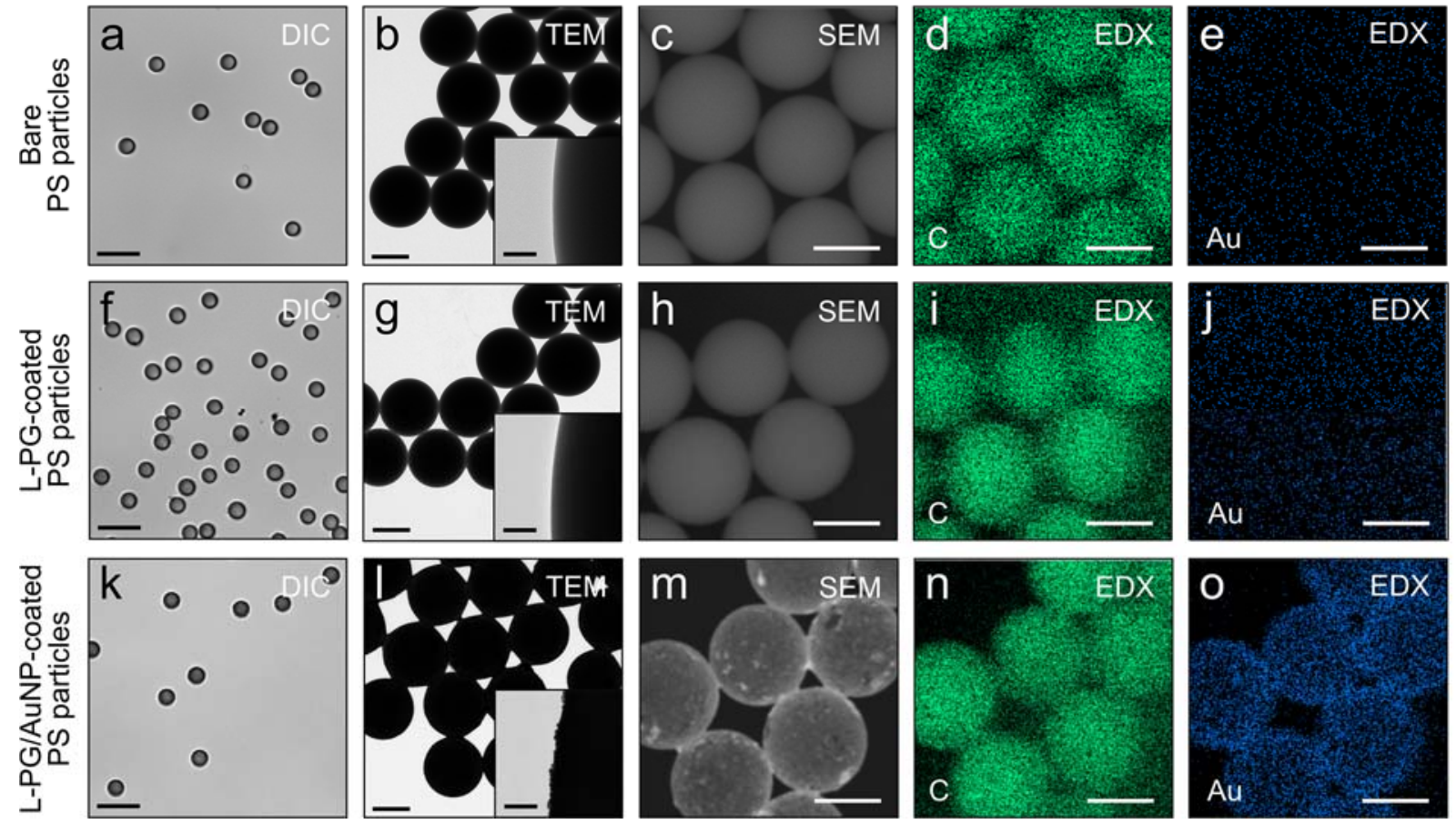

Fig. 4. PS microparticles coated with L-PG and L-PG/AuNP LbL assemblies. (a, $f, k)$ Differential interference contrast (DIC) microscopy, (b, $g$, I) TEM, and $(c, h, m)$ SEM images, and $(d, i, n) C$ and $(e, j, o)$ Au EDX mapping of bare PS microparticles (a-e), L-PG-coated PS microparticles ( $f-$ j), and 3-bilayer L-PG/AuNP-coated PS microparticles (k-0). Scale bars: DIC $(10 \mu \mathrm{m})$, TEM $(2 \mu \mathrm{m})$ and inset $(100 \mathrm{~nm})$, and SEM and EDX (3 $\mu \mathrm{m})$.

QCM-D was used to monitor the kinetics of the hybrid film assembly process by depositing sequential layers of L-PG and AuNPs. L-PG first directly adhered to the gold substrate acting as a primer layer, followed by the deposition of AuNPs on the L-PG layer to form a hybrid bilayer structure, stabilized by disulfide-gold interactions (Figure 3b). Both L-PG and AuNP deposition reached equilibrium within 30 min of incubation. The dissipation factor increased when an L-PG layer was deposited, indicating that the L-PG layer was distorted during crystal oscillations and therefore displayed characteristics of a soft film (Figure S13). In contrast, the dissipation factor remained constant upon deposition of AuNPs, showing that the rigid AuNPs followed the oscillation of the crystal without being deformed. These results demonstrated the hybrid nature of the coating.

The film growth was also monitored by AFM measurements of the average dry thickness and roughness of the films. After the deposition of each bilayer, the dry thickness and roughness increased by $6.3 \pm 0.6$ and $2.4 \pm 0.1 \mathrm{~nm}$, respectively, indicating that the films were compact and homogeneous with a high surface coverage (Figure 3c and Figures S14, 15). Importantly, the L-PG/AuNP hybrid films on glass displayed excellent chemical stability up to $60 \mathrm{~min}$ in several harsh chemical environments such as $10 \mathrm{mM}$ PBS (pH 7.4), 1 $\mathrm{M} \mathrm{HCl}, 1 \mathrm{M} \mathrm{HNO}_{3}, 10 \mathrm{mM} \mathrm{NaBH}_{4}, 1 \mathrm{M} \mathrm{H}_{2} \mathrm{O}_{2}$, $n$-hexane, EtOH, $17 \mathrm{mM}$ SDS, and $10 \mathrm{mM} \mathrm{GSH}$ (Figure 3d). Notably, the stability of the multilayered film was significantly higher than that of the single-layer L-PG film. This can be ascribed to the shielding effect of the AuNP layer, ${ }^{35}$ which prevents the detachment and degradation of L-PG.

Remarkably, the L-PG/AuNP hybrid film formed on various materials such as rigid (PS, PMMA, PP, polyether ether ketone) and flexible (PTFE, PET, Parafilm, Viton, polyurethane) polymeric, oxide (glass), and metal (Ni foam) substrates (Figure $3 e$ and Figure S16). The deposition is indicated by the change in color of the substrates upon adsorption of AuNPs mediated by interactions with the primer L-PG layer. The coating formed on the different substrates remained stable at room temperature $\left(25^{\circ} \mathrm{C}\right)$ for up to 6 months in dried state. To further extend the possible use of the L-PG/AuNP hybrid film to microscale objects, PS microparticles $(3.2 \mu \mathrm{m})$ were successfully coated with three bilayers of L-PG/AuNP (Figure 4). Disperse bare PS microparticles (Figure 4a) displayed, a smooth particle edge (Figure $4 \mathrm{~b}$ and inset) and a homogeneous distribution of carbon only (Figure $4 c-e)$. The PS microparticles coated with one layer of the L-PG film did not show any aggregation (Figure 4f). Similarly to the bare PS 
microparticles, the L-PG-coated PS microparticles displayed a smooth particle edge (Figure 4g and inset) and a uniform distribution of carbon only (Figure $4 \mathrm{~h}-\mathrm{j}$ ). The PS microparticles coated with three bilayers of L-PG/AuNP LbL assemblies did not show evidence of aggregation (Figure 4k). However, TEM images showed an increased contrast and roughness in the edge of the particles, compared with those of the bare PS and L-PG-coated PS microparticles, which was attributed to the presence of the dense AuNPs (Figure $4 \mathrm{l}$ and inset). Furthermore, the SEM images and EDX mapping showed the homogeneous presence of both carbon and gold in the coating (Figure $4 \mathrm{~m}-\mathrm{o}$ ). These results further confirmed the presence of AuNPs and therefore the successful preparation of L-PG/AuNP LbL assemblies onto PS microparticles.

\section{Catalytic and SERS properties of hybrid LbL assemblies}

To demonstrate the applications of the tunable hybrid films, a chemical reactor was designed by coating the inner wall of PP tubes with L-PG/AuNP bilayers for catalysis. The catalytic activities of the hybrid films were evaluated through the $\mathrm{NaBH}_{4}$-mediated reduction of methylene blue (MB) to leucomethylene blue (LMB) in the presence of metal catalysts. The reaction kinetics was monitored via UV-vis spectroscopy in real time, wherein changes in the absorption peak at $664 \mathrm{~nm}$, corresponding to $\mathrm{MB}$, were monitored as $M B$ was converted to colorless $L M B$. When an MB solution containing $\mathrm{NaBH}_{4}$ was incubated in an L-PG/AuNP-coated PP tube, the blue solution turned transparent over time, indicating the successful reduction reaction (Figure 5a). In contrast, the bare and L-PG-coated PP tubes showed no reduction of $\mathrm{MB}$ (Figure S17). Our results show that the catalytic efficacy of the system relies on the layers of the AuNPs coating, and faster reduction kinetics were obtained in reactors with a higher number of L-PG/AuNP bilayers (Figure $5 b$ ). Specifically, MB reduction in the reactors followed a second-order reaction with rate constants of $6.0 \pm 0.8,11.0 \pm 0.8$, and $20 \pm 2 \mathrm{mM}^{-1} \mathrm{~min}^{-1}$ for 1,3 , and 5 L-PG/AuNPs bilayers, respectively. This improvement was due to the increasing overall amount of AuNPs and easy access of small molecules into the L-PG matrix. Notably, the catalysts in the film showed high stability because of the negligible amount of AuNPs leached into the solution during the reaction (Figure $5 \mathrm{c}$ ). The robust assembly of these hybrid films will allow defining functional coatings on various materials for catalytic purposes.

SERS can be used to detect vibrational fingerprints of chemicals with high sensitivity, wherein the Raman signals of targets are significantly augmented when located near plasmonic nanostructures (e.g., AgNPs) owing to the enhanced electromagnetic field arising from the excitation of LSPR bands. Thus, by integrating AgNPs into the coatings, we can generate a SERS-active surface on glass substrates that can be used to monitor the concentration of chemical species in the surrounding. Using CV as a model, we found that the hybrid coating of three L-PG/AgNP bilayers displayed SERS activity toward CV (Figure 5d). The signal of CV at $1615 \mathrm{~cm}^{-1}$ increased with increasing $\mathrm{CV}$ concentration ranging from $500 \mathrm{nM}$ to $10 \mu \mathrm{M}$ (Figure S18). A limit of detection of $60 \mathrm{nM}$ (signal-to-noise ratio of 3) was achieved. The facile preparation of the present hybrid coatings is expected to enable the design of "smart" SERS-active coatings on diverse substrates for sensing applications.
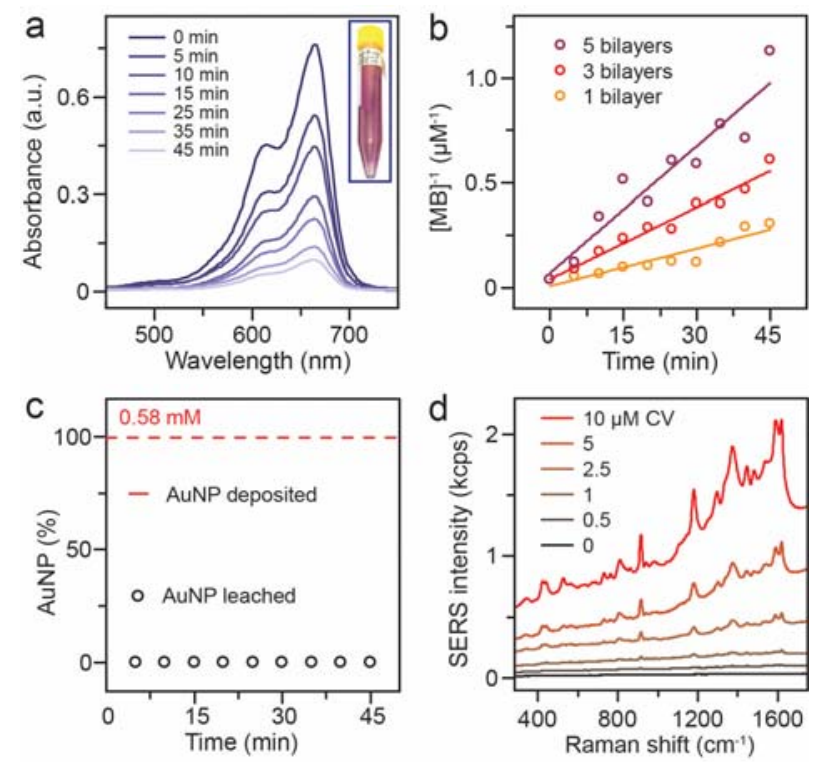

Fig. 5. Catalysis and sensing applications of $L-P G /$ metal nanoparticles $\mathrm{LbL}$ assemblies. (a) UV-Vis spectra of $M B$ recorded during its degradation via $\mathrm{NaBH}_{4}$ in a 3-bilayer L-PG/AuNP-coated PP tube and photograph (inset) of the functionalized tube. (b) $\mathrm{NaBH}_{4}$-mediated MB degradation kinetics in 1-, 3-, and 5-bilayer L-PG/AuNP-coated PP tubes. (c) Comparison of the amount of AuNPs leached from a 3bilayer L-PG/AuNP-coated PP tube and the total amount of AuNPs originally deposited in the same tube. (d) SERS spectra of a 3-bilayer L-PG/AgNP-coated glass as a function of different concentrations of CV.

\section{Conclusions}

In the present work, adhesive glycogen nanoparticles were used as building blocks to prepare hybrid multifunctional films on various substrates, including organic and inorganic materials, in an aqueous environment. The adhesive and functional properties of L-PG are ascribed to the hydrophilic-hydrophobic interactions between the amphiphilic L-PG and the substrate. These polysaccharide-derived nano building blocks displayed negligible cytotoxicity, and the L-PG-based films were stable in harsh chemical environments but biodegradable. Furthermore, the presence of disulfide moieties onto L-PG allowed the integration of diverse metal nanoparticles to prepare multilayered films. As proof of concept, hybrid functional coatings were developed and applied for the catalysis and SERSbased detection of analytes. The PG-based coatings and multilayered films provide a versatile platform for engineering interfaces with modular functionalities and potential uses in nanomedicine, material science, catalysis, and sensing.

\section{Conflicts of interest}

There are no conflicts to declare.

\section{Acknowledgements}


This work was funded by an Australian Research Council (ARC) Future Fellowship (F. Cavalieri, FT140100873) and a National Health and Medical Research Council Senior Principal Research Fellowship (F. Caruso, GNT1135806) schemes. This project received funding from the European Union Horizon 2020 research and innovation program under the Marie Skłodowska-Curie grant agreement No. 690901 ("Nanosupremi") and No. 798565 (A. Amodio "RE-IMMUNE"). This work was performed at the ARC Centre of Excellence in Convergent Bio-Nano Science and Technology (project number CE140100036) and in part at the Materials Characterization and Fabrication Platform (MCFP) at The University of Melbourne.

\section{Notes and references}

(1) M. Bjornmalm, K. J. Thurecht, M. Michael, A. M. Scott and F. Caruso, ACS Nano, 2017, 11, 9594.

(2) M. Holzinger, A. Le Goff and S. Cosnier, Front. Chem., 2014, 2, 63.

(3) S. Goenka, V. Sant and S. Sant, J. Controlled Rel., 2014, 173, 75.

(4) D. Lu, J. Zhou, S. Hou, Q. Xiong, Y. Chen, K. Pu, J. Ren and H. Duan, Adv. Mater., 2019, 31, 1902733.

(5) X. Wang, J. Pu, B. An, Y. Li, Y. Shang, Z. Ning, Y. Liu, F. Ba, J. Zhang and C. Zhong, Adv. Mater., 2018, 30, 1705968.

(6) R. Liu, J. Zhao, Q. Han, X. Hu, D. Wang, X. Zhang and P. Yang, Adv. Mater., 2018, 30, 1802851.

(7) P. P. Tomanin, P. V. Cherepanov, Q. A. Besford, A. J. Christofferson, A. Amodio, C. F. McConville, I. Yarovsky, F. Caruso and F. Cavalieri, ACS Appl. Mater. Interfaces, 2018, 10, 42786.

(8) Z. Shi, K. Neoh, E. Kang, C. Poh and W. Wang, J. Biomed. Mater. Res., Part A, 2008, 86, 865.

(9) V. K. Thakur and M. K. Thakur, ACS Sustainable Chem. Eng., 2014, 2, 2637.

(10) T. Nishimura and K. Akiyoshi, Wiley Interdiscip. Rev.: Nanomed. Nanobiotechnol., 2017, 9, e1423.

(11) C. Wang, R. A. Venditti and K. Zhang, Appl. Microbiol. Biotechnol., 2015, 99, 5791.

(12) S. E. Burke and C. J. Barrett, Biomacromolecules, 2003, 4, 1773.

(13) T. I. Croll, A. J. O'Connor, G. W. Stevens and J. J. Cooper-White, Biomacromolecules, 2006, 7, 1610.

(14) S. G. Ball and M. K. Morell, Annu. Rev. Plant Biol., 2003, 54, 207. (15) P. J. Roach, A. A. Depaoli-Roach, T. D. Hurley and V. S. Tagliabracci, Biochem. J., 2012, 441, 763.

(16) Q. A. Besford, F. Cavalieri and F. Caruso, Adv. Mater., 2019, 1904625, DOI: 10.1002/adma.201904625.

(17) M. Grossutti and J. R. Dutcher, Biomacromolecules, 2016, 17, 1198.

(18) J. D. Nickels, J. Atkinson, E. Papp-Szabo, C. Stanley, S. O. Diallo, S. Perticaroli, B. Baylis, P. Mahon, G. Ehlers and J. Katsaras, Biomacromolecules, 2016, 17, 735.

(19) M. Wojnilowicz, A. Glab, A. Bertucci, F. Caruso and F. Cavalieri, ACS Nano, 2018, 13, 187.

(20) M. Wojnilowicz, Q. A. Besford, Y.-L. Wu, X. J. Loh, J. A. Braunger, A. Glab, C. Cortez-Jugo, F. Caruso and F. Cavalieri, Biomaterials, 2018, 176, 34.

(21) M. Perrone, A. Lopalco, A. Lopedota, A. Cutrignelli, V. Laquintana, J. Douglas, M. Franco, E. Liberati, V. Russo and S. Tongiani, Eur. J. Pharm. Biopharm., 2017, 119, 161.

(22) R. Kandimalla, S. Dash, A. C. Bhowal, S. Kalita, N. C. Talukdar, S. Kundu and J. Kotoky, Int. J. Nanomed., 2017, 12, 7025.

(23) F. Lu, A. Mencia, L. Bi, A. Taylor, Y. Yao and H. HogenEsch, J. Controlled Rel., 2015, 204, 51.
(24) Q. A. Besford, M. Wojnilowicz, T. Suma, N. Bertleff-Zieschang, F. Caruso and F. Cavalieri, ACS Appl. Mater. Interfaces, 2017, 9, 16869. (25) S. Aasen, A. Pospisilova, T. Eichler, J. Panek, M. Hruby, P. Stepanek, E. Spriet, D. Jirak, K. Skaftnesmo and F. Thorsen, Int. J. Mol. Sci., 2015, 16, 21658.

(26) Y. Zhu, W. Tong and C. Gao, Soft Matter, 2011, 7, 5805.

(27) B. Macphail and M. A. Brook, Greem Chem., 2017, 19, 4373.

(28) J. Li, D. Maniar, X. Qu, H. Liu, C.-Y. Tsao, E. Kim, W. E. Bentley, C. Liu and G. F. Payne, Biomacromolecules, 2019, 20, 969.

(29) Y. Xie and Y. Yao, Carbohydr. Polym., 2018, 180, 29.

(30) M. Rabyk, M. Hruby, M. Vetrik, J. Kucka, V. Proks, M. Parizek, R. Konefal, P. Krist, D. Chvatil and L. Bacakova, Carbohydr. Polym., 2016, 152, 271.

(31) P. Patra, A. P. Rameshbabu, D. Das, S. Dhara, A. B. Panda and S. Pal, Polym. Chem., 2016, 7, 5426.

(32) M. Bertoldo, G. Zampano, L. Suffner, E. Liberati and F. Ciardelli, Polym. Chem., 2013, 4, 653.

(33) T. Liebert, M. A. Hussain, M. N. Tahir and T. Heinze, Polym. Bull., 2006, 57, 857.

(34) F. Gu, C. Hu, Z. Tai, C. Yao, J. Tian, L. Zhang, Q. Xia, C. Gong, Y. Gao and S. Gao, Sci. Rep., 2016, 6, 36281.

(35) P. Thoniyot, M. J. Tan, A. A. Karim, D. J. Young and X. J. Loh, Adv. Sci., 2015, 2, 1400010. 